\title{
Inhibition of phosphoinositide 3-kinase/Akt pathway decreases hypoxia inducible factor-1 $\alpha$ expression and increases therapeutic efficacy of paclitaxel in human hypoxic gastric cancer cells
}

\author{
JING ZHANG, HUA GUO, JIN-SHUI ZHU, YU-CHEN YANG, WEI-XIONG CHEN and NI-WEI CHEN \\ Department of Gastroenterology, Shanghai Jiao Tong University Affiliated Sixth People's Hospital, \\ Shanghai 200233, P.R. China
}

Received August 18, 2013; Accepted February 20, 2014

DOI: 10.3892/ol.2014.1963

\begin{abstract}
The phosphatidylinositol-3-kinase (PI3K)/Akt signaling pathway plays an important role in cell proliferation, transformation, apoptosis, tumor growth and angiogenesis. Paclitaxel is commonly used to treat multiple human malignancies; however, the underlying mechanisms of paclitaxel in gastric cancer (GC) have not been fully investigated. In the present study, specimens from $45 \mathrm{GC}$ and 36 chronic gastritis patients were collected, and the correlations of PI3K, phosphorylated-Akt (p-Akt) and hypoxia-inducible factor-1 $\alpha$ (HIF-1 $\alpha$ ) expression with the clinicopathological characteristics of GC were analyzed by immunohistochemistry. The human SGC-7901 GC cells under hypoxic conditions were pretreated with the PI3K inhibitor, LY294002 $(40 \mu \mathrm{M})$, and paclitaxel $(0.1 \mu \mathrm{M})$. The expression levels of PI3K, p-Akt and HIF- $1 \alpha$ were detected by quantitative polymerase chain reaction and western blotting. Cell proliferative activity and apoptosis were evaluated by the Cell Counting Kit- 8 assay and flow cytometry. As a result, the rates of positive expression of PI3K, p-Akt and HIF-1 $\alpha$ were significantly higher in GC compared with chronic gastritis patients (each $\mathrm{P}<0.01$ ), and were positively associated with the tumor-node-metastasis (TNM) staging, lymph node metastases, lymphatic infiltration and vascular infiltration (each $\mathrm{P}<0.01$ ), but inversely correlated with tumor differentiation $(\mathrm{P}<0.01)$ in patients with $\mathrm{GC}$. Under hypoxic conditions, the combined inhibition of the PI3K/Akt pathway with paclitaxel markedly reduced the proliferative activity and induced cell apoptosis in GC cells compared with the single treatment of PI3K inhibitor or paclitaxel (each $\mathrm{P}<0.01$ ), and was accompanied by a decreased expression of HIF-1 $\alpha$. Overall, our findings indicate that the increased
\end{abstract}

Correspondence to: Professor Jin-Shui Zhu, Department of Gastroenterology, Shanghai Jiao Tong University Affiliated Sixth People's Hospital, 600 Yishan Road, Shanghai 200233, P.R. China E-mail: zhujs1803@163.com; jing5522724@163.com

Key words: phosphoinositide 3-kinase, Akt, hypoxia inducible factor- $1 \alpha$, gastric cancer, paclitaxel, hypoxia expression of the PI3K/Akt/HIF-1 $\alpha$ pathway was closely correlated with tumor differentiation, TNM staging, lymph node metastases and lymphatic and vascular infiltration. The inhibition of the PI3K/Akt pathway enhanced the therapeutic efficacy of paclitaxel in GC cells under hypoxic conditions, suggesting that the PI3K/Akt/HIF-1 $\alpha$ pathway may act as an important therapeutic target for paclitaxel treatment of GC.

\section{Introduction}

Gastric cancer (GC) is one of the most common types of malignancies worldwide, with an estimated 934,000 cases reported globally in 2002, and is the second most common cause of cancer-related mortality (1). GC is also a genetic disease developing from a multistep process. Single or multiple mutations in genes associated with growth control, invasion and metastasis, form the molecular genetic basis of malignant transformation and tumor progression (2). Chemotherapy, to a certain extent, plays a critical role in the treatment of malignant tumors. However, the identification of predictive biomarkers of resistance or sensitivity to chemotherapy remains a fundamental challenge in the selection of patients most likely to benefit from it.

The phosphatidylinositol-3-kinase (PI3K)/Akt pathway has been shown to be activated in a variety of cancer types. Studies have shown that the increased expression of PI3K or phosphoinositide 3-Akt (p-Akt) contributes to gallbladder carcinogenesis (3) or predicts the survival of advanced endometrial carcinoma (4). Activation of the PI3K/Akt pathway is required for the apoptotic evasion (5) and is significantly associated with increasing tumor grade, decreased apoptosis and clinical outcome in human gliomas (6). It supports the development of metastatic cancer and promotes the aggressive behavior of soft tissue sarcoma $(7,8)$, indicating the PI3K/Akt pathway as an important biomarker for the prognosis of cancer patients.

Hypoxia-inducible factor-1 $\alpha$ (HIF-1 $\alpha$ ) plays an essential role in the adaptive response of cells to hypoxia and is associated with aggressive tumor behavior. HIF-1 $\alpha$ is highly expressed in small-cell lung cancer and aids in predicting the overall survival of patients (9), as well as in selecting patients most likely to benefit from HIF-1 $\alpha$-targeted therapies (10). 
HIF-1 $\alpha$ is also overexpressed in mantle cell lymphoma, where it enhances the aggressive potential and, therefore, this observation may result in more efficient target therapies (11). Notably, hypoxia induces a biphasic effect on HIF-1 $\alpha$ stabilization with accumulation in early hypoxia, depending on activation of the PI3K/Akt pathway (12). In hypoxic tumor cells, reactive oxygen species increase HIF-1 $\alpha$ transcription via the PI3K/Akt pathway (13), and silencing of HIF-1 $\alpha$ suppresses tumorigenicity of renal cell carcinoma through the regulation of the PI3K/Akt pathway (14). Activation of PI3K/Akt signaling promotes the progression of hepatocarcinogenesis, while its blockade controls angiogenesis and tumor growth by regulating the expression of HIF-1 $\alpha(15,16)$.

Notably, the expression of PI3K/Akt is increased in non-small cell lung cancer treated with adjuvant chemotherapy and serves as a novel independent prognostic biomarker (17). Deregulation of the PI3K/Akt pathway is associated with resistance to the chemotherapeutic agent and confers drug resistance to treatment with paclitaxel in breast cancer $(18,19)$. Although certain studies have demonstrated the enhanced effectiveness of targeting tumor cells with combinations of chemotherapeutic agents and signal transduction inhibitors (20), the enhancing effects of blockade of the PI3K/Akt pathway on paclitaxel in hypoxic GC cells remains unclear. In the present study, the correlations of PI3K, p-Akt and HIF-1 $\alpha$ expression with the clinicopathological characteristics of patients with GC were analyzed. Hypoxic GC SGC-7901 cells were pretreated with LY294002 and/or paclitaxel to investigate the enhancing effects of the PI3K inhibitor on paclitaxel through cell proliferation activity and apoptosis.

\section{Materials and methods}

Materials. The human GC SGC7901 cell line was donated from the Shanghai Tumor Research Institute (no. 01842; Shanghai, China). The primers of PI3K and HIF-1 $\alpha$ were synthesized by the Shanghai Biological Engineering Technology Co., Ltd. (Shanghai, China). The PI3K rabbit-anti-human polyclonal antibody (sc-1637) was purchased from Santa Cruz Biotechnology, Inc. (Santa Cruz, CA, USA); p-Akt rabbit-anti-human polyclonal antibody (D9E) and $\beta$-actin were purchased from Cell Signaling Technology, Inc. (Danvers, MA, USA); and HIF-1 $\alpha$ rabbit-anti-human polyclonal antibody (BA0912) was obtained form Wuhan Boster Biological Engineering Co., Ltd (Wuhan, China). The GC tissue samples treated with paclitaxel were obtained from severe combined immune deficiency (SCID) mice orthotopically implanted with human GC cells from the Gastrointestinal Disease Laboratory of Shanghai Sixth People's Hospital (Shanghai, China).

Drugs and reagents. Paclitaxel was donated by Professor G-Y Fan from the Kunming Botany Institute (Yunnan, China); the Cell Counting Kit-8 (CCK-8) was purchased from Tongren Chemical Institute (Japan); Dulbecco's modified Eagle's medium (DMEM) and fetal bovine serum (FBS) were purchased from Thermo Fisher Scientific Inc. (Waltham, MA, USA); TRIzol ${ }^{\circledR}$ reagent and LY294002 were obtained from Invitrogen Life Technologies (Carlsbad, CA, USA);
M-MLV Reverse Transcriptase was purchased from Promega Corporation (Madison, WI, USA); SYBR Green master mix was purchased from Takara Bio, Inc., (Otsu, Japan); the Annexin V-fluorescein isothiocyanate cell apoptosis detection kit was obtained from KeyGen Biotech., Co., Ltd. (ab14085; Nanjing, China); and the Enhanced Chemiluminescence (ECL)-PLUS ${ }^{\mathrm{TM}}$ western blotting reagents were purchased from GE Healthcare (Piscataway, NJ, USA).

Clinical samples and data. A total of 45 patients with GC and 36 patients with chronic gastritis were enrolled in this study at the General Surgery and Digestive Endoscopy Room from December, 2007 to June, 2008. The pathological staging was determined according to the American Joint Committee on Cancer tumor-node-metastasis (TNM) staging system. The use of the tissue samples and clinical data was approved by the Medical Ethics Committee of Shanghai Jiao Tong University (Shanghai, China).

Immunohistochemical analysis. The protein expression of PI3K, p-Akt and HIF-1 $\alpha$ were analyzed by immunohistochemical staining. The anti-PI3K, p-Akt and HIF-1 $\alpha$ antibodies were used at 1:100 dilutions. Endogenous peroxidase was inhibited by incubation with freshly prepared $3 \%$ hydrogen peroxide with $0.1 \%$ sodium azide. Non-specific staining was blocked with $0.5 \%$ casein and $5 \%$ normal serum. The tissues were incubated with biotinylated antibodies and horseradish peroxidase (Cell Signaling Technology, Inc.). Staining was developed with diaminobenzidine substrate and sections were counterstained with hematoxylin and eosin. Normal serum or phosphate-buffered saline (PBS) replaced the antibodies in negative controls. The images were analyzed with Image-Pro Plus 4.5 System (Media Cybernetics, Inc., Rockville, MD, USA). The total optical density value and area of intracellular fluorescence for each section was measured.

Cell culture and pretreatment. The SGC7901 cells were cultured in DMEM supplemented with $10 \%$ heat-inactivated FBS, $100 \mathrm{U} / \mathrm{ml}$ penicillin and $100 \mu \mathrm{g} / \mathrm{ml}$ streptomycin. The cells were stored in a humidified atmosphere of $5 \% \mathrm{CO}_{2}$ at $37^{\circ} \mathrm{C}$ for $30 \mathrm{~min}$. Under normal oxygen, the cells were incubated in $20 \% \mathrm{O}_{2}$ and $5 \% \mathrm{CO}_{2}$ with saturated humidity at $37^{\circ} \mathrm{C}$ for $30 \mathrm{~min}$. The hypoxic cells were incubated in an AnaeroPack ${ }^{\mathrm{TM}}$ containing $20 \% \mathrm{CO}_{2}$ and $<1 \% \mathrm{O}_{2}$ at $37^{\circ} \mathrm{C}$ for $30 \mathrm{~min}$. The cells pretreated under hypoxic conditions were further treated with the PI3K inhibitor, LY294002, (40 Mm) for $30 \mathrm{~min}$, followed by paclitaxel $(0.1 \mathrm{Mm})$.

Quantitative polymerase chain reaction ( $q P C R)$. To quantitatively determine the mRNA expression levels of PI3K and HIF-1 $\alpha$ in GC SGC-7901 cells, qPCR was used. Total RNA of each clone was extracted with TRIzol reagent according to the manufacturer's instructions. Reverse-transcription using M-MLV, and cDNA amplification using SYBR Green master mix kit, were performed according to the manufacturer's protocol. The genes were amplified using specific oligonucleotide primers and a human $\beta$-actin gene was used as an endogenous control.The PCR primersequences wereas follows: Forward, 5'-AAGAAGCAAGCAGCTGAG-3' and reverse, 5'-CTACAGAGCAGGCATAG-3' for PI3K; forward, 5'-TCA 
AAGTCGGACAGCCTC-3' and reverse, 5'-CCAGCAGTC TACATGC-3' for HIF-1 $\alpha$; forward, 5'-CTTCGAGCAAGA GATGGC-3' and reverse, 5'-CTCCTTCTGCATCCTGTC-3' for $\beta$-actin. Data were analyzed using the comparative $\mathrm{Ct}$ method $\left(2^{-\Delta \Delta \mathrm{Ct}}\right)$. Three separate experiments were performed for each clone.

Western blot analysis. The hypoxic SGC-7901 cells treated with LY294002 and/or paclitaxel were harvested and extracted using lysis buffer [Tris- $\mathrm{HCl}$, sodium-dodecyl sulfate (SDS), mercaptoethanol and glycerol] purchased from Santa Cruz Biotechnology, Inc. Cell extracts were heated to boiling point for $5 \mathrm{~min}$ in loading buffer and equal amounts of cell extracts were separated on 15\% SDS-PAGE gels. Separated protein bands were transferred into polyvinylidene fluoride membranes, which were blocked in 5\% skimmed milk powder. The primary antibodies against PI3K, p-Akt and HIF-1 $\alpha$ were diluted according to the manufacturer's instructions and incubated overnight at $4^{\circ} \mathrm{C}$. Horseradish peroxidase-linked secondary biotinylated antibodies were added at a dilution ratio of 1:1,000 and incubated at room temperature for $2 \mathrm{~h}$. The membranes were washed with PBS three times and the immunoreactive bands were visualized using the ECL-PLUS/Kit according to the manufacturer's instructions. The relative protein levels in different cell lines were normalized to the GAPDH concentration. Three separate experiments were performed for each clone. Finally, the immune complexes were developed using an ECL detection kit according to the manufacturer's instructions (ECL GST western blotting detection kit, Pierce Biotechnology, Inc., Waltham, MA, USA) and the GelGDoc2000 imaging system (Bio-Rad Laboratories GmbH, Munich, Germany) was employed to analyze the bands, and the protein levels by the relative optical density.

CCK-8 assay. Cell proliferation following treatment with LY294002 and/or paclitaxel was measured by the CCK-8 assay. The GC SGC-7901 cells were seeded at a density of $2 \times 10^{4}$ cells $/ 100 \mu 1 /$ well in 96 -well plates and left to attach overnight. The medium was then removed and $200 \mu 1$ of FBS was added followed by LY294002 and/or paclitaxel. The cells were incubated under these conditions for 24, 48, 72, 96, 120 and $148 \mathrm{~h}$ at $37^{\circ} \mathrm{C}$ in a humidified atmosphere of $5 \% \mathrm{CO}_{2}$. After the designated time, CCK- 8 was added to each well containing $200 \mu \mathrm{l}$ of the culture medium and the oligopeptide mixture, and further incubated for $4 \mathrm{~h}$ at $37^{\circ} \mathrm{C}$. The amount of formazan dye was measured at $450 \mathrm{~nm}$ using the Multi-Mode Microplate Reader (Nanchang Biotek Medical Device Co., Ltd., Nanchang, China). All the experiments were performed in triplicate and repeated three times.

Cell apoptosis analysis. Cell apoptosis detection was performed using the BD Accuri ${ }^{\mathrm{TM}}$ C6 Flow cytometer (BD Biosciences, San Jose, CA, USA). The exposure of PBS on the extracellular side of the cell membrane was quantified by propidium iodide (PI) staining (Invitrogen Life Technologies). The SGC-7901 cells were placed in six-well plates and, after $24 \mathrm{~h}$ of incubation, the cells were treated with LY294002 and/or paclitaxel for $24 \mathrm{~h}$ and then harvested. Following centrifugation, cell pellets were washed twice with
Table I. Expression of PI3K, p-AKT and HIF-1 $\alpha$ in gastric cancer (Gray values).

\begin{tabular}{llclc}
\hline Markers & \multicolumn{1}{c}{ Group } & Cases & Gray value & P-value \\
\hline PI3K & Gastric cancer & 45 & $202.4 \pm 10.7$ & $<0.01$ \\
& Chronic gastritis & 36 & $220.3 \pm 4.3$ & \\
p-AKT & Gastric cancer & 45 & $223.6 \pm 11.7$ & $<0.01$ \\
& Chronic gastritis & 36 & $234.0 \pm 4.6$ & \\
HIF-1 $\alpha$ & Gastric cancer & 45 & $200.3 \pm 6.9$ & $<0.01$ \\
& Chronic gastritis & 36 & $218.7 \pm 4.4$ & \\
\hline
\end{tabular}

PI3K, phosphatidylinositol-3-kinase; p-AKT, phosphorylated-Akt; HIF- $1 \alpha$, hypoxia-inducible factor- $1 \alpha$.

cold PBS. The cells were then incubated with $5 \mu 1$ of PI at room temperature for $15 \mathrm{~min}$ in the dark. Following incubation, $400 \mu \mathrm{l}$ of $1 \mathrm{X}$ binding buffer was added to each tube. The cells were immediately analyzed by flow cytometry.

Statistical analysis. Data are expressed as the means \pm standard deviation where applicable. Statistically significant differences in each assay were determined by SPSS software, version 20.0 (SPSS, Inc., Chicago, IL, USA). Differences in each group were tested for significance using Student's t-test or one-way analysis of variance. $\mathrm{P}<0.05$ was considered to indicate a statistically significant difference.

\section{Results}

Correlations of PI3K, $p$-Akt and HIF-1 $\alpha$ expression with the clinicopathological characteristics. The GC tissue sections were analyzed by immunohistochemistry (IHC) and Image-Pro Plus 4.5 software (Media Cybernetics, Inc.). As shown in Fig. 1 and Table I, the positive expression of PI3K, p-Akt and HIF-1 $\alpha$ was predominantly localized in the cytoplasm of the GC tissue cells, but was not identified in the chronic gastritis cells. The expression intensities of PI3K, p-Akt and HIF-1 $\alpha$ were significantly increased in GC tissues compared with chronic gastritis tissues (each $\mathrm{P}<0.01$ ).

The correlations of PI3K, p-Akt and HIF-1 $\alpha$ expression and various clinical and pathological characteristics were analyzed. As summarized in Table II, no significant correlation was found between PI3K, p-Akt and HIF-1 $\alpha$ expression and gender, age, tumor size and peripheral nerve infiltration in patients with GC (each $\mathrm{P}>0.05$ ), while their expression was significantly correlated with TNM staging, lymph node metastases, lymphatic infiltration and vascular infiltration (each $\mathrm{P}<0.01$ ), but inversely correlated with tumor differentiation $(\mathrm{P}<0.01)$.

Effects of LY294002 and/or paclitaxel on the expression of PI3K, $p$-Akt and HIF-1 $\alpha$. qPCR and western blot analysis were performed to detect the effects of LY294002 and paclitaxel on the expression of PI3K, p-Akt and HIF-1 $\alpha$ in the GC SGC-7901 cells. LY294002 combined with paclitaxel markedly inhibited 
Table II. Correlation of PI3K, p-AKT and HIF-1 $\alpha$ expression with the clinicopathological characteristics of patients with GC.

\begin{tabular}{|c|c|c|c|c|c|c|c|}
\hline Variables & Cases & PI3K & $\mathrm{P}$-value & p-AKT & P-value & HIF- $1 \alpha$ & P-value \\
\hline \multicolumn{8}{|l|}{ Age, years } \\
\hline$\leq 68$ & 23 & $201.0 \pm 9.2$ & \multirow[t]{2}{*}{0.052} & $223.7 \pm 10.7$ & \multirow[t]{2}{*}{0.841} & $200.7 \pm 6.8$ & \multirow[t]{2}{*}{0.335} \\
\hline$>68$ & 22 & $203.9 \pm 10.3$ & & $223.4 \pm 11.7$ & & $199.8 \pm 7.0$ & \\
\hline \multicolumn{8}{|l|}{ Gender } \\
\hline Male & 32 & $202.9 \pm 10.9$ & \multirow[t]{2}{*}{0.279} & $223.9 \pm 11.0$ & \multirow[t]{2}{*}{0.526} & $200.7 \pm 6.6$ & \multirow[t]{2}{*}{0.142} \\
\hline Female & 13 & $201.2 \pm 10.4$ & & $223.0 \pm 11.7$ & & $199.2 \pm 7.4$ & \\
\hline \multicolumn{8}{|l|}{ Tumor size, $\mathrm{cm}$} \\
\hline$\leq 5$ & 30 & $202.4 \pm 8.4$ & \multirow[t]{2}{*}{0.926} & $224.2 \pm 10.8$ & \multirow[t]{2}{*}{0.256} & $200.8 \pm 5.4$ & \multirow[t]{2}{*}{0.184} \\
\hline$>5$ & 15 & $202.9 \pm 8.4$ & & $200.3 \pm 6.9$ & & $199.3 \pm 9.1$ & \\
\hline \multicolumn{8}{|c|}{ Degree of differentiation } \\
\hline Well/moderately & 15 & $206.3 \pm 10.9$ & \multirow[t]{2}{*}{$<0.01$} & $227.6 \pm 10.6$ & \multirow[t]{2}{*}{$<0.01$} & $202.1 \pm 6.3$ & \multirow[t]{2}{*}{0.005} \\
\hline Poorly & 30 & $200.4 \pm 10.2$ & & $221.6 \pm 10.9$ & & $199.4 \pm 7.0$ & \\
\hline \multicolumn{8}{|l|}{ TNM staging } \\
\hline I+II & 20 & $207.7 \pm 8.8$ & \multirow[t]{2}{*}{$<0.01$} & $228.6 \pm 9.5$ & \multirow[t]{2}{*}{$<0.01$} & $204.3 \pm 5.6$ & \multirow[t]{2}{*}{$<0.01$} \\
\hline III+IV & 25 & $198.1 \pm 10.3$ & & $219.6 \pm 10.8$ & & $197.1 \pm 6.1$ & \\
\hline \multicolumn{8}{|c|}{ Lymph node metastases } \\
\hline No & 19 & $208.7 \pm 8.4$ & \multirow[t]{2}{*}{$<0.01$} & $228.7 \pm 9.6$ & \multirow[t]{2}{*}{$<0.01$} & $204.3 \pm 5.7$ & \multirow[t]{2}{*}{$<0.01$} \\
\hline Yes & 26 & $197.8 \pm 10.2$ & & $219.8 \pm 10.7$ & & $197.3 \pm 6.1$ & \\
\hline \multicolumn{8}{|c|}{ Lymphatic vessel infiltration } \\
\hline- & 11 & $207.6 \pm 7.0$ & \multirow[t]{2}{*}{$<0.01$} & $230.7 \pm 8.4$ & \multirow[t]{2}{*}{$<0.01$} & $205.7 \pm 5.3$ & \multirow[t]{2}{*}{$<0.01$} \\
\hline+ & 34 & $200.7 \pm 11.2$ & & $221.3 \pm 11.0$ & & $198.5 \pm 6.4$ & \\
\hline \multicolumn{8}{|l|}{ Vascular infiltration } \\
\hline- & 29 & $205.0 \pm 9.9$ & \multirow[t]{2}{*}{$<0.01$} & $226.6 \pm 10.6$ & \multirow[t]{2}{*}{$<0.01$} & $201.6 \pm 7.2$ & $<0.01$ \\
\hline+ & 16 & $197.6 \pm 10.4$ & & $218.1 \pm 10.0$ & & $197.9 \pm 5.5$ & \\
\hline Perineural infiltrati & & & & & & & \\
\hline- & 6 & $203.6 \pm 6.6$ & 0.324 & $225.7 \pm 10.4$ & 0.265 & $202.5 \pm 4.8$ & 0.053 \\
\hline+ & 39 & $202.2 \pm 11.3$ & & $223.2 \pm 11.3$ & & $199.9 \pm 7.1$ & \\
\hline
\end{tabular}

PI3K, phosphatidylinositol-3-kinase; p-AKT, phosphorylated-Akt; HIF-1 $\alpha$, hypoxia-inducible factor-1 $\alpha$; TNM, tumor-node-metastasis.

Table III. Proliferative activity of gastric cancer cells (optical density values).

\begin{tabular}{|c|c|c|c|c|c|c|}
\hline \multirow[b]{2}{*}{ Groups } & \multicolumn{6}{|c|}{ Day } \\
\hline & 1 & 2 & 3 & 4 & 5 & 6 \\
\hline Normoxia & $0.45 \pm 0.01$ & $0.75 \pm 0.02$ & $1.25 \pm 0.06$ & $1.60 \pm 0.04$ & $1.86 \pm 0.04$ & $2.12 \pm 0.10$ \\
\hline Normoxia+paclitaxel & $0.43 \pm 0.02$ & $0.60 \pm 0.03^{\mathrm{a}}$ & $0.87 \pm 0.0^{\mathrm{a}}$ & $1.22 \pm 0.04^{\mathrm{a}}$ & $1.54 \pm 0.05^{\mathrm{a}}$ & $1.74 \pm 0.07^{\mathrm{a}}$ \\
\hline Hypoxia & $0.46 \pm 0.03$ & $0.68 \pm 0.04^{\mathrm{a}}$ & $1.05 \pm 0.05^{\mathrm{a}}$ & $1.40 \pm 0.07^{\mathrm{a}}$ & $1.65 \pm 0.02^{\mathrm{a}}$ & $1.85 \pm 0.04^{\mathrm{a}}$ \\
\hline Hypoxia+paclitaxel & $0.44 \pm 0.06$ & $0.59 \pm 0.04^{\mathrm{a}, \mathrm{b}}$ & $0.83 \pm 0.05^{\mathrm{a}, \mathrm{b}}$ & $1.11 \pm 0.05^{\mathrm{a}, \mathrm{b}}$ & $1.38 \pm 0.07^{\mathrm{a}, \mathrm{b}}$ & $1.60 \pm 0.10^{\mathrm{a}, \mathrm{b}}$ \\
\hline Hypoxia+LY294002 & $0.45 \pm 0.04$ & $0.64 \pm 0.03^{\mathrm{a}-\mathrm{c}}$ & $0.85 \pm 0.02^{\mathrm{a}-\mathrm{c}}$ & $1.10 \pm 0.10^{\mathrm{a}, \mathrm{b}}$ & $1.33 \pm 0.12^{\mathrm{a}-\mathrm{c}}$ & $1.61 \pm 0.11^{\mathrm{a}-\mathrm{c}}$ \\
\hline Hypoxia+paclitaxel+LY294002 & $0.42 \pm 0.07$ & $0.53 \pm 0.03^{\mathrm{a}-\mathrm{c}}$ & $0.67 \pm 0.03^{\mathrm{a}-\mathrm{c}}$ & $0.87 \pm 0.03^{\mathrm{a}-\mathrm{c}}$ & $1.11 \pm 0.12^{\mathrm{a}-\mathrm{c}}$ & $1.29 \pm 0.04^{\mathrm{a}-\mathrm{c}}$ \\
\hline
\end{tabular}

${ }^{\mathrm{a}} \mathrm{P}<0.01$, vs. normoxia; ${ }^{\mathrm{P}}<0.01$, vs. hypoxia; ${ }^{\mathrm{C}} \mathrm{P}<0.01$, vs. hypoxia+paclitaxel.

the mRNA (Fig. 2A and B) expression levels of PI3K and HIF-1 $\alpha$ (it was not necessary to detect the expression of $\mathrm{p}$-Akt as it is downstream of PI3K) and protein (Fig. 3A and B) expression levels of PI3K, p-Akt and HIF-1 $\alpha$ in GC SGC-7901 cells compared with the single treatment of LY294002 or paclitaxel (each P<0.01). LY294002 or paclitaxel decreased the expression of PI3K, p-Akt and HIF-1 $\alpha$ at the transcriptional and translational levels compared with the hypoxic group (each $\mathrm{P}<0.01$ ). 


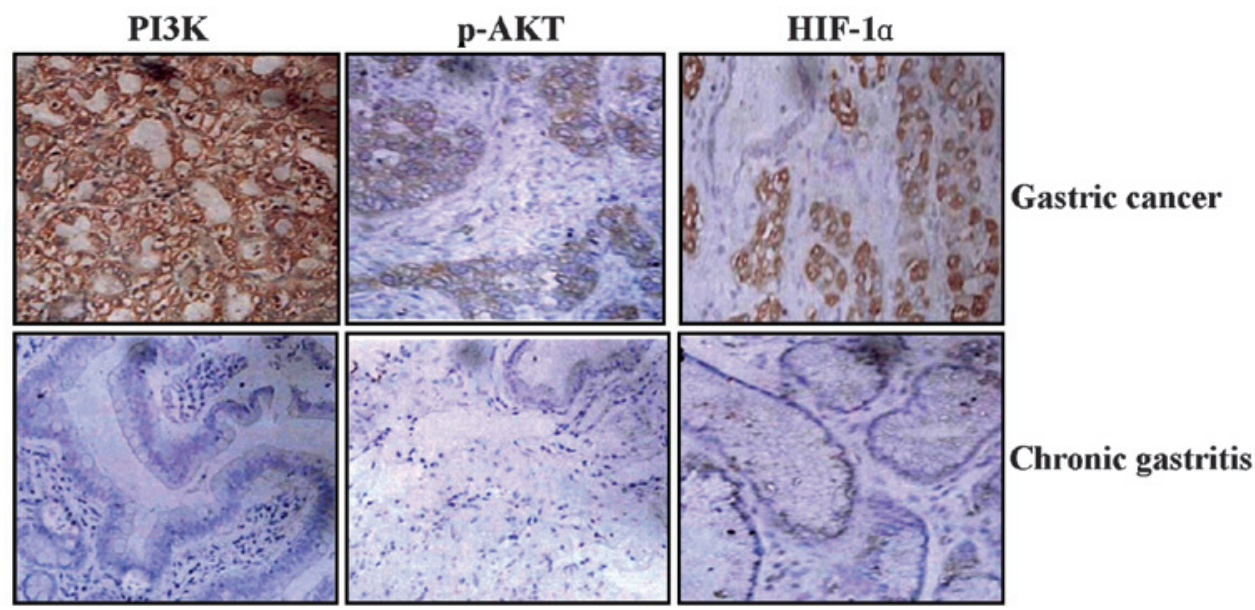

Figure 1. Expression of PI3K, p-AKT and HIF-1 $\alpha$ in GC tissues (magnification, x200). The positive expression of PI3K, p-AKT and HIF-1 $\alpha$ was predominantly localized in the cytoplasm of GC tissue cells but was rarely identified in chronic gastritis, and their expression intensities were significantly increased in GC tissue compared with chronic gastritis tissue. PI3K, phosphatidylinositol-3-kinase; p-AKT, phosphorylated-Akt; HIF-1 $\alpha$, hypoxia-inducible factor-1 $\alpha$; GC, gastric cancer.

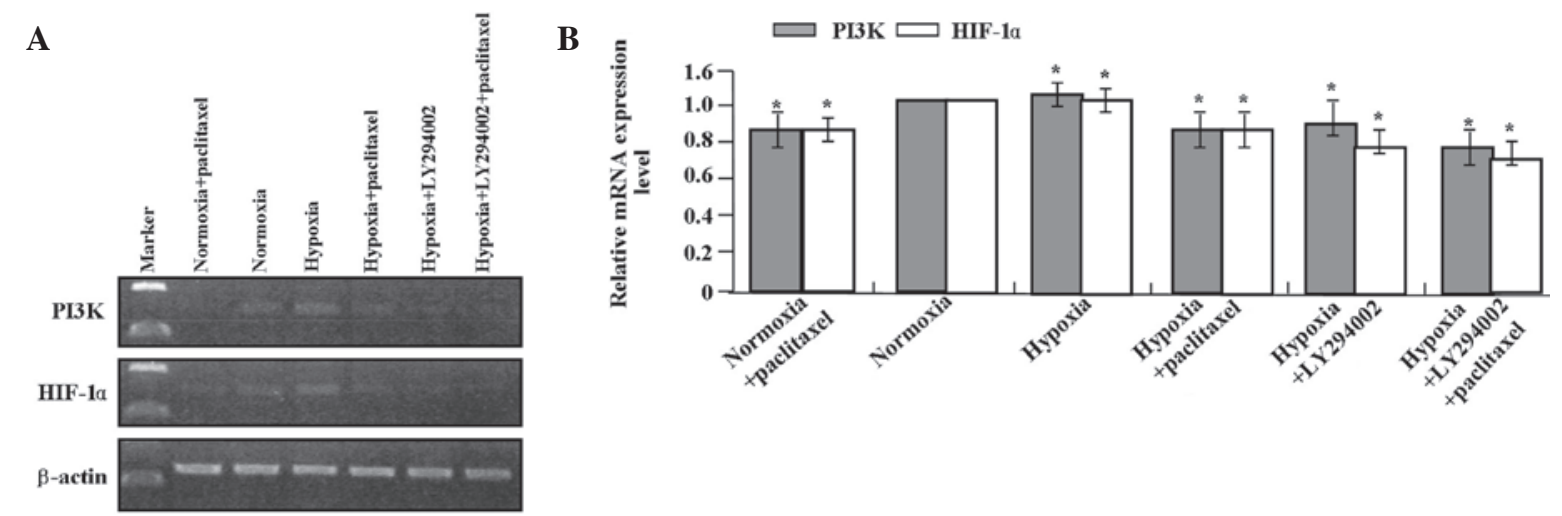

Figure 2. Effects of LY294002 and/or paclitaxel on the mRNA expression of PI3K and HIF-1 $\alpha$. (A) Quantitative polymerase chain reaction was performed to detect the mRNA expression levels. (B) LY294002 combined with paclitaxel markedly reduced the mRNA expression levels of PI3K and HIF-1 $\alpha$ compared with the single treatment of LY294002 or paclitaxel (each P<0.01). LY294002 or paclitaxel decreased the RNA expression levels of PI3K and HIF-1 $\alpha$ compared with the hypoxia group. ${ }^{*} \mathrm{P}<0.01$ compared with normoxia. PI3K, phosphatidylinositol-3-kinase; HIF-1 $\alpha$, hypoxia-inducible factor-1 $\alpha$.
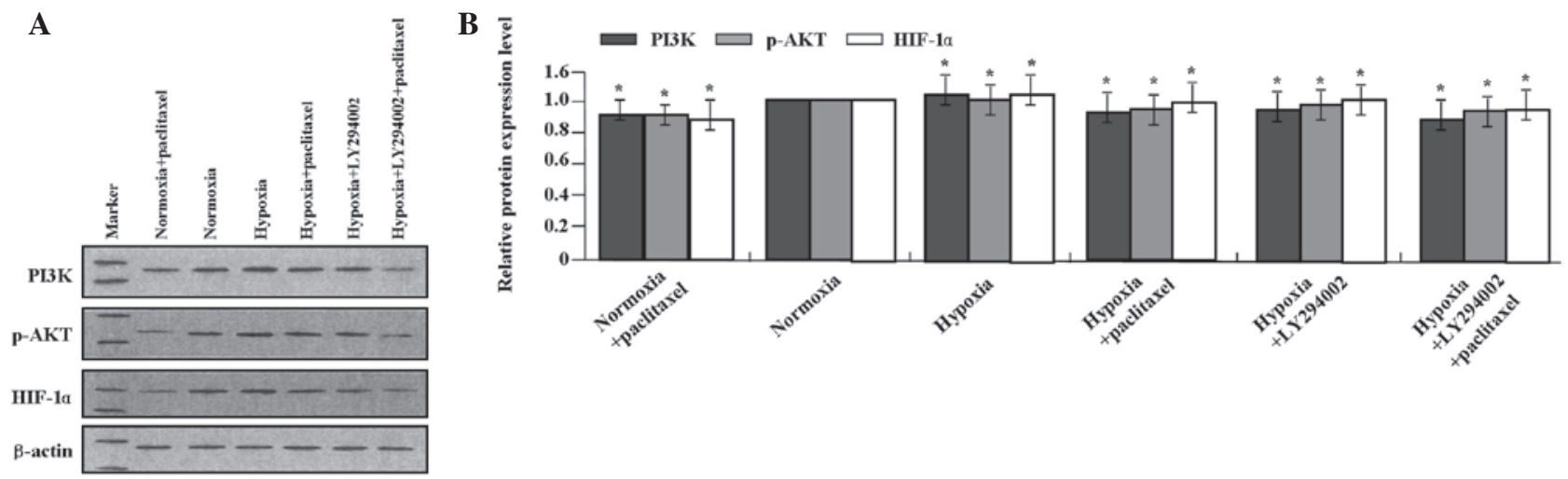

Figure 3. Effects of LY294002 and/or paclitaxel on the protein expression of PI3K, p-AKT and HIF-1 $\alpha$. (A) Western blotting was performed to detect the protein expression levels. (B) LY294002 combined with paclitaxel significantly decreased the protein expression levels of PI3K, p-AKT and HIF-1 $\alpha$ compared with the single treatment of LY294002 or paclitaxel (each P<0.01). LY294002 or paclitaxel decreased the protein expression of PI3K, p-AKT and HIF-1 $\alpha$ compared with the hypoxia group. " $\mathrm{P}<0.01$ compared with normoxia. PI3K, phosphatidylinositol-3-kinase; p-AKT, phosphorylated-Akt; HIF-1 $\alpha$, hypoxia-inducible factor-1 $\alpha$.

Effects of LY294002 and/or paclitaxel on cell proliferation. To determine whether LY294002 and/or paclitaxel affect the proliferative activity of hypoxic GC cells, the CCK-8 assay was performed to detect cell viability. As summarized in Table III, LY294002 combined with paclitaxel significantly decreased the cell viability in SGC-7901 cells compared with the single 
A
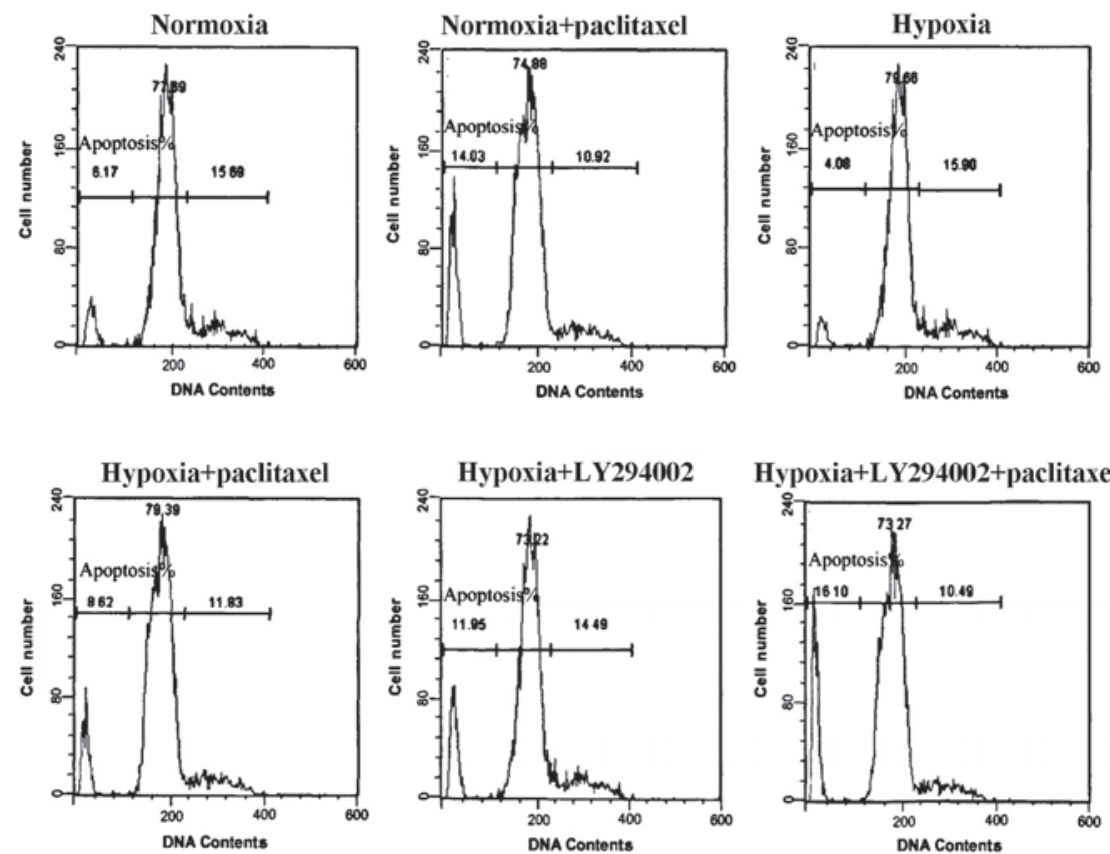

DNA Contents

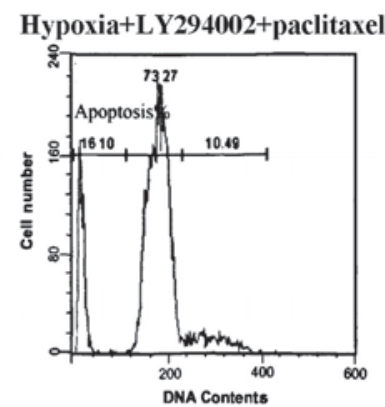

B

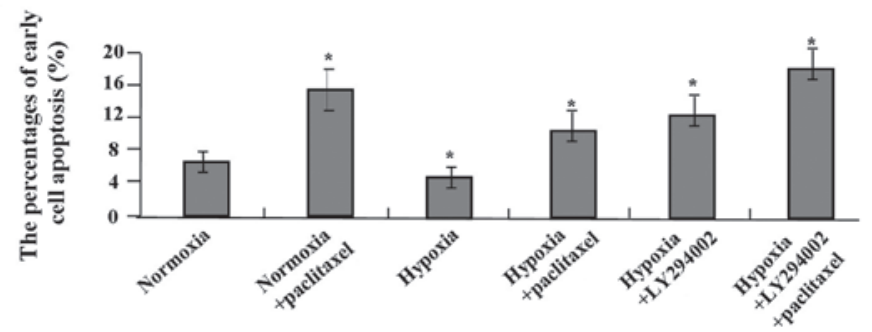

Figure 4. Effects of LY294002 and/or paclitaxel on cell apoptosis. (A) Cell apoptosis was measured by flow cytometry analysis using propidium iodide staining. (B) Following treatment with LY294002 and/or paclitaxel for $24 \mathrm{~h}$, LY294002 combined with paclitaxel significantly increased the percentage of early cell apoptosis compared with the single treatment of LY294002 or paclitaxel (each P<0.01). LY294002 or paclitaxel also improved the percentage of early cell apoptosis compared with the hypoxia group. ${ }^{*} \mathrm{P}<0.01$ compared with normoxia.

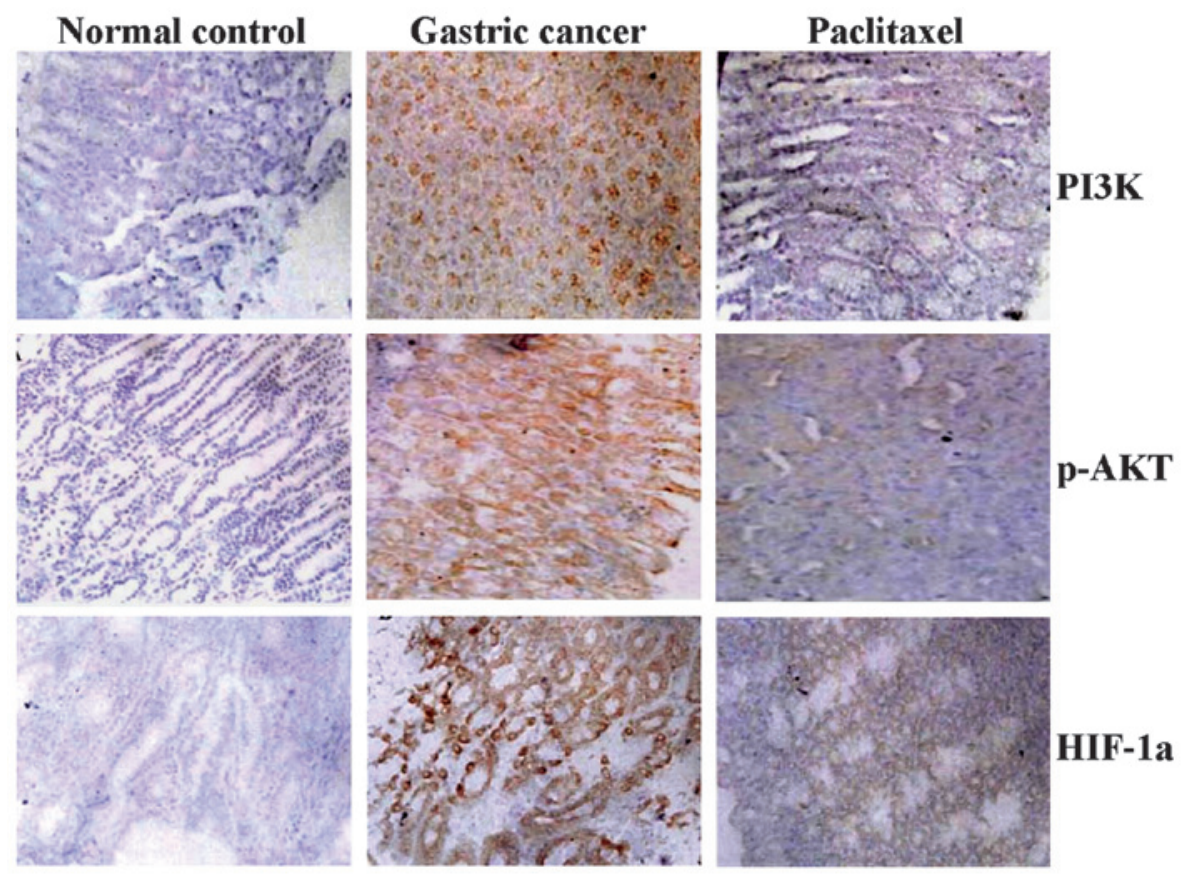

Figure 5. Effects of paclitaxel on the expression of PI3K, p-AKT and HIF-1 $\alpha$ in vivo. The expression of PI3K, p-AKT and HIF-1 $\alpha$ were assessed by immunohistochemistry in severe combined immune deficiency mice orthotopically implanted with human GC tissues and treated with paclitaxel. Paclitaxel decreased the expression of PI3K, p-AKT and HIF-1 $\alpha$ compared with the GC and normal control groups. PI3K, phosphatidylinositol-3-kinase; p-AKT, phosphorylated-Akt; HIF-1 $\alpha$, hypoxia-inducible factor- $1 \alpha$. 
treatment of LY294002 or paclitaxel (each $\mathrm{P}<0.01$ ). In addition, LY294002 or paclitaxel reduced cell viability compared with the hypoxic group (each $\mathrm{P}<0.01)$.

Effects of LY294002 and/or paclitaxel on cell apoptosis. Cell apoptosis was measured by flow cytometry using PI staining. Following treatment with LY294002 and/or paclitaxel for $24 \mathrm{~h}$, LY294002 combined with paclitaxel significantly increased the percentage of early cell apoptosis compared with the single treatment of LY294002 or paclitaxel (each P<0.01) (Fig. 4A and B). LY294002 or paclitaxel increased the percentage of early cell apoptosis compared with the hypoxic group (both $\mathrm{P}<0.01$ ).

Effects of paclitaxel on the expression of PI3K, p-Akt and HIF-1 $\alpha$ in vivo. SCID mice orthotopically implanted with human GC tissues were treated with paclitaxel (intraperitoneal administration of $5 \mathrm{mg} / \mathrm{kg}$ ) and the effects of paclitaxel on the expression of PI3K, p-Akt and HIF-1 $\alpha$ were assessed by IHC. Paclitaxel decreased the expression of PI3K, p-Akt and HIF-1 $\alpha$ (Fig. 5) compared with the GC and normal control groups (each $\mathrm{P}<0.01$ ).

\section{Discussion}

The PI3K/Akt pathway is one of the most important signaling networks in cancer. Emerging evidence indicates that activation of this pathway plays a significant role in the development and progression of certain malignancies. Zhang et al reported that the PI3K/Akt pathway is expressed in 71.7 (43/60) and 68.3\% $(41 / 60)$ of colon cancer and is closely associated with serous coat infiltration and lymphatic metastasis (21), serving as an independent prognostic marker for patients with colorectal cancer (CRC) (22). HIF-1 $\alpha$ is a transcription factor recognized to control the delivery of oxygen and nutrients through the induction of angiogenesis under hypoxic conditions. Overexpression of HIF-1 $\alpha$ is significantly correlated with histology, depth of invasion and poor prognosis for patients with GC, and may be utilized for tumor-specific molecular target-based therapy (23). In the present study, the positive expression of PI3K, p-Akt and HIF-1 $\alpha$ were significantly increased in GC tissues compared with chronic gastritis, and were associated with TNM staging, lymph node metastases and lymphatic and vascular infiltration, but inversely correlated with tumor differentiation. This suggested that PI3K, p-Akt and HIF-1 $\alpha$ are potential therapeutic targets for GC.

Inhibiting the molecules involved in the PI3K/Akt or HIF-1 $\alpha$ signal transduction pathway is a possible strategy for the treatment of cancer. The PI3K inhibitor and conventional chemotherapy provide an effective approach to inhibiting tumor growth in ovarian cancer (24). Paclitaxel is extensively used in chemotherapy for various cancers. PI3K is involved in low susceptibility of CRC to paclitaxel and PI3K-targeting agents may enable a new paclitaxel-based chemotherapy for CRC (25). Furthermore, the Akt inhibitor increases the therapeutic efficacy of paclitaxel for patients with ovarian cancer (26). HIF-1 $\alpha$ affects the sensitivity of paclitaxel in lung cancer cells and targeted inhibition of HIF-1 $\alpha$ may overcome the drug resistance of paclitaxel (27). However, an individual study has reported that pharmacological PI3K/Akt inhibition antagonizes the efficacy of chemotherapeutic agents primarily effective in the
S or G2 phase of the cell cycle (28). Our current study indicated that combining the PI3K inhibitor, LY294002, with paclitaxel significantly decreased cell viability and increased cell apoptosis in hypoxic GC SGC-7901 cells compared with the single treatment of LY294002 or paclitaxel. Moreover, treatment with LY294002 or paclitaxel reduced cell viability and induced apoptosis compared with the hypoxic GC cells, suggesting that PI3K/Akt-targeted therapy with paclitaxel is more efficacious for treating GC with activated PI3K/Akt signaling.

Notably, the PI3K/Akt pathway regulates HIFa activity (29), and HIF-1 $\alpha$-dependent transcription is blocked by negative Akt or PI3K and by the wild-type phosphatase and tensin homolog (30). Direct evidence has also revealed that activation of the PI3K/Akt/HIF-1 $\alpha$ pathway plays a critical role in mediating hypoxia-induced drug resistance resulting in an unfavorable treatment outcome of hepatocellular carcinoma (31). However, Manohar et al (32) reported that the HIF-1 $\alpha$ inhibitor modulates the PI3K/Akt pathway and contributes to antitumor activity. In the present study, LY294002 combined with paclitaxel markedly inhibited the expression of PI3K, p-Akt and HIF-1 $\alpha$ in GC SGC-7901 cells compared with the single treatment of LY294002 or paclitaxel, and LY294002 or paclitaxel decreased the expression of PI3K and p-Akt in hypoxic conditions, suggesting that targeting PI3K/Akt signaling in tumor cells may inhibit HIF-1 $\alpha$ expression and increase the therapeutic efficacy of paclitaxel.

In conclusion, our findings suggest that the increased expression of the PI3K/Akt or HIF-1 $\alpha$ pathway is closely correlated with tumor differentiation, TNM staging, lymph node metastases, and lymphatic and vascular infiltration. In addition, inhibition of the PI3K/Akt pathway enhanced the therapeutic efficacy of paclitaxel in GC cells under hypoxic conditions, suggesting that the PI3K/Akt or HIF-1 $\alpha$ pathway may serve as an important therapeutic target for the paclitaxel treatment of cancer.

\section{Acknowledgements}

This study was supported by the National Nature Science Foundation of China (grant nos. 81272752 and 81302093).

\section{References}

1. Jemal A, Bray F, Center MM, et al: Global cancer statistics. CA Cancer J Clin 61: 69-90, 2011.

2. Tajima Y, Yamazaki K, Makino R, et al: Gastric and intestinal phenotypic marker expression in early differentiated-type tumors of the stomach: clinicopathologic significance and genetic background. Clin Cancer Res 12: 6469-6479, 2006.

3. Li Q and Yang Z: Expression of phospho-ERK1/2 and PI3-K in benign and malignant gallbladder lesions and its clinical and pathological correlations. J Exp Clin Cancer Res 28: 65, 2009.

4. Uegaki K, Kanamori Y, Kigawa J, et al: PTEN-positive and phosphorylated-Akt-negative expression is a predictor of survival for patients with advanced endometrial carcinoma. Oncol Rep 14: 389-392, 2005.

5. Sun ZJ, Chen G, Hu X, et al: Activation of PI3K/Akt/IKK-alpha /NF-kappaB signaling pathway is required for the apoptosis-evasion in human salivary adenoid cystic carcinoma: its inhibition by quercetin. Apoptosis 15: 850-863, 2010.

6. Chakravarti A, Zhai G, Suzuki Y, et al: The prognostic significance of phosphatidylinositol 3-kinase pathway activation in human gliomas. J Clin Oncol 22: 1926-1933, 2004.

7. Xue G, Restuccia DF, Lan Q, et al: Akt/PKB-mediated phosphorylation of Twist1 promotes tumor metastasis via mediating cross-talk between PI3K/Akt and TGF- $\beta$ signaling axes. Cancer Discov 2: 248-259, 2012. 
8. Valkov A, Kilvaer TK, Sorbye SW, et al: The prognostic impact of Akt isoforms, PI3K and PTEN related to female steroid hormone receptors in soft tissue sarcomas. J Transl Med 9: 200, 2011.

9. Lee GW, Go SI, Cho YJ, et al: Hypoxia-inducible factor-1 $\alpha$ and excision repair cross-complementing 1 in patients with small cell lung cancer who received front-line platinum-based chemotherapy: a retrospective study. J Thorac Oncol 7: 528-534, 2012.

10. Park S, Ha SY, Cho HY, et al: Prognostic implications of hypoxia-inducible factor- $1 \alpha$ in epidermal growth factor receptor-negative non-small cell lung cancer. Lung Cancer 72 : 100-107, 2011

11. Argyriou P, Papageorgiou SG, Panteleon V, et al: Hypoxia-inducible factors in mantle cell lymphoma: implication for an activated mTORC $1 \rightarrow$ HIF-1 $\alpha$ pathway. Ann Hematol 90 315-322, 2011.

12. Mottet D, Dumont V, Deccache Y, et al: Regulation of hypoxia-inducible factor-1alpha protein level during hypoxic conditions by the phosphatidylinositol 3-kinase/Akt/glycogen synthase kinase 3beta pathway in HepG2 cells. J Biol Chem 278 : 31277-31285, 2003.

13. Koshikawa N, Hayashi J, Nakagawara A and Takenaga $\mathrm{K}$ : Reactive oxygen species-generating mitochondrial DNA mutation up-regulates hypoxia-inducible factor-1alpha gene transcription via phosphatidylinositol 3-kinase-Akt/protein kinase C/histone deacetylase pathway. J Biol Chem 284: 33185-33194, 2009.

14. Xu K, Ding Q, Fang Z, et al: Silencing of HIF-1alpha suppresses tumorigenicity of renal cell carcinoma through induction of apoptosis. Cancer Gene Ther 17: 212-222, 2010.

15. TanakaH,YamamotoM,Hashimoto N, etal:Hypoxia-independent overexpression of hypoxia-inducible factor 1alpha as an early change in mouse hepatocarcinogenesis. Cancer Res 66 : 11263-11270, 2006.

16. Fang J, Ding M, Yang L, et al: PI3K/PTEN/AKT signaling regulates prostate tumor angiogenesis. Cell Signal 19: 2487-2497, 2007.

17. Shi Y, Chen L, Li J, et al: Prognostic and predictive values of pERK1/2 and pAkt-1 expression in non-small cell lung cancer patients treated with adjuvant chemotherapy. Tumour Biol 32: 381-390, 2011.

18. McCubrey JA, Steelman LS, Abrams SL, et al: Roles of the RAF/MEK/ERK and PI3K/PTEN/AKT pathways in malignant transformation and drug resistance. Adv Enzyme Regul 46: 249-279, 2006

19. Liang K, Lu Y, Li X, et al: Differential roles of phosphoinositide-dependent protein kinase-1 and akt1 expression and phosphorylation in breast cancer cell resistance to Paclitaxel, Doxorubicin, and gemcitabine. Mol Pharmacol 70: 1045-1052, 2006.
20. Abrams SL, Steelman LS, Shelton JG, et al: Enhancing therapeutic efficacy by targeting non-oncogene addicted cells with combinations of signal transduction inhibitors and chemotherapy. Cell Cycle 9: 1839-1846, 2010.

21. Zhang Y, Liu X, Zhang J, et al: The expression and clinical significance of PI3K, pAkt and VEGF in colon cancer. Oncol Lett 4: 763-766, 2012.

22. Kim JG, Chae YS, Sohn SK, et al: Clinical significance of genetic variations in the $\mathrm{PI} 3 \mathrm{~K} / \mathrm{PTEN} / \mathrm{AKT} / \mathrm{mTOR}$ pathway in Korean patients with colorectal cancer. Oncology 79: 278-282, 2010.

23. Isobe T, Aoyagi K, Koufuji K, et al: Clinicopathological significance of hypoxia-inducible factor-1 alpha (HIF-1 $\alpha$ ) expression in gastric cancer. Int J Clin Oncol 18: 293-304, 2013.

24. Hu L, Hofmann J, Lu Y, et al: Inhibition of phosphatidylinositol 3'-kinase increases efficacy of paclitaxel in in vitro and in vivo ovarian cancer models. Cancer Res 62: 1087-1092, 2002.

25. Xu R, Nakano K, Iwasaki H, et al: Dual blockade of phosphatidylinositol 3'-kinase and mitogen-activated protein kinase pathways overcomes paclitaxel-resistance in colorectal cancer. Cancer Lett 306: 151-160, 2011.

26. Kim SH, Juhnn YS and Song YS: Akt involvement in paclitaxel chemoresistance of human ovarian cancer cells. Ann N Y Acad Sci 1095: 82-89, 2007.

27. Zeng L, Kizaka-Kondoh S, Itasaka S, et al: Hypoxia inducible factor-1 influences sensitivity to paclitaxel of human lung cancer cell lines under normoxic conditions. Cancer Sci 98: 1394-1401, 2007.

28. Fekete M, Santiskulvong C, Eng C and Dorigo O: Effect of PI3K/Akt pathway inhibition-mediated G1 arrest on chemosensitization in ovarian cancer cells. Anticancer Res 32: 445-452, 2012.

29. Shafee N, Kaluz S, Ru N and Stanbridge EJ: PI3K/Akt activity has variable cell-specific effects on expression of HIF target genes, CA9 and VEGF, in human cancer cell lines. Cancer Lett 282: 109-115, 2009.

30. Zhong H, Chiles K, Feldser D, et al: Modulation of hypoxia-inducible factor 1alpha expression by the epidermal growth factor/phosphatidylinositol 3-kinase/PTEN/AKT/FRAP pathway in human prostate cancer cells: implications for tumor angiogenesis and therapeutics. Cancer Res 60: 1541-1545, 2000.

31. Jiao $\mathrm{M}$ and Nan KJ: Activation of PI3 kinase/Akt/HIF-1a pathway contributes to hypoxia-induced epithelial-mesenchymal transition and chemoresistance in hepatocellular carcinoma. Int J Oncol 40: 461-468, 2012.

32. Manohar SM, Padgaonkar AA, Jalota-Badhwar A, et al: A novel inhibitor of hypoxia-inducible factor-1 $\alpha$ P3155 also modulates PI3K pathway and inhibits growth of prostate cancer cells. BMC Cancer 11: 338, 2011 